\title{
Three-Body Recombination in Ultracold Systems: Prediction of Weakly-Bound Atomic Trimer Energies
}

\author{
Lauro Tomio $^{1 *}$, Victo S. Filho ${ }^{1}$, M. T. Yamashita ${ }^{2}$, A. Gammal ${ }^{2}$, \\ and T. Frederico ${ }^{3}$

\footnotetext{
${ }^{1}$ Instituto de Física Teórica, Universidade Estadual Paulista, 01405-900, São Paulo, Brasil

2 Laboratório do Acelerador Linear, Instituto de Física da USP, 05315-970, São Paulo, Brasil

${ }^{3}$ Departamento de Física, Instituto Tecnológico de Aeronáutica, CTA, 12228-900, São José dos Campos, Brasil
}

\begin{abstract}
The three-body recombination coefficient of a trapped ultracold atomic system, together with the corresponding two-body scattering length $a$, allow us to predict the energy $E_{3}$ of the shallow trimer bound state, using a universal scaling function. The production of dimers in Bose-Einstein condensates, from three-body recombination processes, in the regime of short magnetic pulses near a Feshbach resonance, is also studied in line with the experimental observation.
\end{abstract}

\section{Introduction}

The interaction among neutral atoms is equivalent to a range zero in the limit of $a \rightarrow \infty$. For identical bosonic atoms, either the Efimov effect [1] or the Thomas collapse 2] can happen when the ratio between $a$ and the range goes to infinity. This is the scaling limit, where the three-boson properties can be determined just by two scales: the dimer binding energy (or virtual dimer energy) and the shallow trimer binding energy [3].

Recently, Bose-Einstein condensation (BEC) has been obtained with trapped ultra-cold atomic gases. Losses of atoms in BEC can occur due to three-atom recombination processes, which are measured in several cases: ${ }^{23} \mathrm{Na} \mid F=1, m_{F}=$ -1) [4, ${ }^{87} \mathrm{Rb}\left|F=1, m_{F}=-1\right\rangle[5,6]$ and ${ }^{85} \mathrm{Rb}\left|F=2, m_{F}=-2\right\rangle[7,8]$. The value of the three-body recombination coefficient, together with the corresponding twobody scattering length, allow us to predict the energy of the shallow trimer bound state for such systems in a trap, as we describe in Sect. 2.

*E-mail: tomio@ift.unesp.br 
Three-body recombination in ultracold systems: Prediction...

Another aspect related to the three-body recombination process, supported by experimental observations, is a possible enhancement due to quantum coherence (laser effect), which may happen in a special situation of fast changes of $a$. In Sect. 3, we discuss the dynamics of BEC related to this aspect of the three-body recombination process. Our conclusions are summarized in Sect. 4.

\section{Weakly bound atomic trimer energies}

It was shown by some of us, in ref. 9], how to obtain the trimer binding energies of a trapped atomic system from the three-body recombination rate and the corresponding two-body scattering length. A universal scaling function which gives the dependence of the recombination parameter as a function of the weakly bound trimer energy $\left(E_{3}\right)$ is calculated within a renormalized scheme for three particles interacting through pairwise Dirac-delta interaction 9 . The value of $E_{3}$ are shown to be in the interval $1<m(a / \hbar)^{2} E_{3}<6.9$ for large $a$. The contribution of a deep-bound state to the prediction, in the case of ${ }^{85} \mathrm{Rb}\left|F=2, m_{F}=-2\right\rangle$, for a particular trap, is shown to be relatively small. Also, using the predicted energies of the triatomic molecules in ultra-cold traps the sizes of the trimers ${ }^{23} N a|1,-1\rangle$ and ${ }^{87} R b|1,-1\rangle$ can be estimated. In this case, a universal scaling function gives the mean-square radii as a function of $E_{3}$ and $a$. The root-meansquare distance between two atoms in a triatomic molecule is estimated to be $0.8 \sqrt{\hbar^{2} /\left(m E_{3}\right)}$, where $m$ is the atomic mass [10].

The rate of three free bosons to recombine, forming a dimer and one remaining particle, is given in the limit of zero energy, by the recombination coefficient [1], 12]

$$
K_{3}=\kappa \frac{\hbar}{m} a^{4}
$$

where $\kappa$ is a dimensionless parameter. When $a>0$, the recombination parameter $\kappa$ oscillates between zero and a maximum value, which is a function of $a$, as shown in refs. 13] $(\kappa \leq 68.4)$, 14] $(\kappa \leq 65)$ and [15] $(\kappa \leq 67.9)$.

By using a general scaling procedure, it is shown in ref. [9] that one can express the functional dependence of $\kappa$ in terms of the two and three-body energies, $\kappa \equiv \kappa\left(\sqrt{E_{2} / E_{3}}\right)$, considering that, for large scattering lengths one has $1 / a=\sqrt{m E_{2} / \hbar^{2}}$. The scaling form of $\kappa$, with explicit $\sqrt{E_{2} / E_{3}}$ dependence, is

$$
\kappa=\kappa_{\max } \sin ^{2}\left(-1.01 \ln \sqrt{\frac{E_{2}}{E_{3}}}+\Delta\left(\sqrt{\frac{E_{2}}{E_{3}}}\right)\right),
$$

where $\Delta\left(\sqrt{E_{2} / E_{3}}\right)=\delta-1.01 \ln \left(\sqrt{m E_{3} / \hbar^{2}}\right)$ and $\delta$ depends on the interaction at short distances.

Next, considering the calculation of the scaling function, with the subtracted form of the Faddeev equations [16, and considering the relation between the transition matrix elements and the three-body recombination coefficient at zero energy, it was derived the functional dependence of $\kappa$ in terms of the ratio $E_{2} / E_{3}$ 
Table 1. For the atomic species ${ }^{A} Z\left|F, m_{F}\right\rangle$, it is shown in the 4 th and 5 th columns the predicted trimer binding energies, in respect to the threshold. $\kappa_{\text {expt }}$ and $E_{2}$ are respectively given in the 2nd and 3rd column. For ${ }^{87} \mathrm{Rb}|1,-1\rangle$, the recombination process was obtained in ref. [5] for noncondensed $\left(^{*}\right)$ and condensed $\left(^{\dagger}\right)$ trapped atoms.

\begin{tabular}{ccccc}
\hline \hline${ }^{A} Z\left|F, m_{F}\right\rangle$ & $\kappa_{\text {expt }}$ & $E_{2}(\mathrm{mK})$ & $S_{3}(\mathrm{mK})$ & $S_{3}^{\prime}(\mathrm{mK})$ \\
\hline${ }^{23} \mathrm{Na}|1,-1\rangle$ & $42 \pm 12$ [4] & 2.85 & 4.9 & 0.21 \\
${ }^{87} \mathrm{Rb}|1,-1\rangle$ & $52 \pm 22^{*}[\underline{5]}$ & 0.17 & 0.39 & 0.005 \\
${ }^{87} \mathrm{Rb}|1,-1\rangle$ & $41 \pm 17^{\dagger}[\underline{5]}$ & 0.17 & 0.30 & 0.013 \\
${ }^{87} \mathrm{Rb}|2,2\rangle$ & $130 \pm 36$ [6] & 0.17 & - & - \\
${ }^{85} \mathrm{Rb}|2,-2\rangle$ & $7.84 \pm 3.4$ [7] [8] & $1.3 \times 10^{-4}$ & $1.14 \times 10^{-4}$ & $3.8 \times 10^{-5}$ \\
\hline \hline
\end{tabular}

(See Fig. 1 of ref. 9]). The scaling limit is well approached in the calculations, with the maximum $\kappa$ occurring at the threshold $\left(E_{3}=E_{2}\right)$ and when $\left(E_{3} / E_{2}\right)^{\frac{1}{2}}=0.38$ [3] such that between two consecutive maximum of $\kappa$ we have $1<m(a / \hbar)^{2} E_{3}<6.9$.

Using the experimental values $\kappa_{\text {expt }}$, for a few atomic species ${ }^{A} Z\left|F, m_{F}\right\rangle$, one can obtain two possible values of the weakly bound triatomic molecular states that are consistent with the universal scaling plot that was derived. The predicted trimer binding energies are given in Table 1, in miliKelvins, in respect to the threshold, $S_{3} \equiv\left(E_{3}-E_{2}\right)$ and $S_{3}^{\prime} \equiv\left(E_{3}^{\prime}-E_{2}\right)$, considering the central values of the experimental dimensionless recombination parameters.

\section{Coherent dimer formation}

One should note that the three-body recombination coefficient was derived in the previous section considering very dilute systems and considering that the twobody interaction is kept fixed. Another interesting possibility may happen in a special situation when we have fast changes of the two-body scattering length $a$. In this case, the three-body recombination process may be enhanced due to quantum coherence. The remaining atoms and dimers are produced in a single state and, due to the symmetrization of the full wave-function, the recombination process which happens in this background is enhanced (laser effect).

Supporting this possibility, it was observed [8] a burst of relatively hot ${ }^{85} \mathrm{Rb}$ atoms with temperature of about $150 \mathrm{nK}$, when a fast magnetic pulse near a Feshbach resonance increases the scattering length up to $4000 a_{0}$. The maximum value of $a$ gives a lower bound for the temperature of the remaining atoms about $75 \mathrm{nK}$ consistent with the experiment. In line with the experimental observation, we make an attempt to describe the remaining number of condensed atoms after the pulse, within a mean field description. For that, we consider that: i) shallow dimers are formed (the temperature of the atoms in the burst is consistent with weakly bound dimers and not deeply bound dimers); ii) the recombination process shows a coherence effect (the burst supports it); and iii) a fitted magnitude of the three-body recombination parameter depends on the rise time $\left(t_{r}\right)$ of the 
scattering length [Also using that $K_{3}$ is proportional to $\left.a(t)^{4}\right]$.

One has to go beyond the mean-field model to implement the coherence effect in a defined theory. The phenomena should be described in a coupled model, with the condensed atoms, the remaining atoms and the dimers. In this respect, it was suggested recently, in a theoretical microscopic approach [17, that in a collision of two atomic condensates producing two molecular condensates in counterpropagating momentum eigenstates, it is possible to appear an atom-molecule laser fed by stimulated three-body recombination processes, where atoms and molecules produced in the same state enhance the three-body recombination rate in respect to the vacuum values.

In an effective way, i.e., using the mean-field equation, the coherence effect (laser effect) will appear as the increase of the magnitude of the recombination parameter in respect to the vacuum value. As the process becomes faster, the loss of coherence of the atoms and dimers is reduced; and, as suggested by our results, the increase in the three-body recombination rate is even higher 18 .

For describing the dynamics of BEC as nonconservative systems, we can follow the extended mean field formalism developed in ref. [19, in which one considers an extended approach of the Gross-Pitaevskii formalism, by including losses of the system by three-body recombination. Here, we should also include the time variation of the relevant physical parameters.

Near a Feshbach resonance, the scattering length $a$ has been observed to vary as a function of magnetic field B, according to theoretical prediction [20, as

$$
a=a_{b} \times\left(1-\frac{\Delta}{B-B_{r}}\right),
$$

where $a_{b}$ is the background scattering length, $B_{r}$ is the resonance magnetic field and $\Delta \equiv\left(B_{0}-B_{r}\right)$ is the resonance width, where $B_{0}$ is the value of $B$ at $a=0$. For the case of ${ }^{85} \mathrm{Rb}$, one has $\Delta \cong 11.0 \mathrm{G}, B_{r} \cong 154.9 \mathrm{G}$ and $a_{b} \cong-450 a_{0}$. We consider in our calculations the same experimental parameters used in ref. [8]: initial field $\mathrm{B}_{0} \cong 166 \mathrm{G}$ (harmonic oscillator state), applied to an initial sample of $\mathrm{N}_{0}=16500$ condensed atoms; spherical symmetry, with mean geometric frequency $\omega \cong 2 \pi \times 12.77 \mathrm{~Hz}$, for simulating the cylindrical geometry of JILA $\left(\omega_{r}=2 \pi \times\right.$ $17.5 \mathrm{~Hz}$ and $\omega_{z}=2 \pi \times 6.8 \mathrm{~Hz}$ ).

For the above conditions, the remaining number of atoms in BEC was obtained and compared with JILA experimental data. The results are given in Table 2. We note that, in order to obtain the best fit of the experimental data, we need to adjust $\kappa$ to values much higher than the vacuum values given by Eq. (11). For instance, if we consider the rise time of the magnetic field pulses lower than $100 \mu \mathrm{s}$, we have $2000 \lesssim \kappa \lesssim 1000$. However, $\kappa$ decreases while $t_{r}$ increases, such that $\kappa \sim 100$ for $t_{r}$ of the order of $250 \mu \mathrm{s}$. In this case, slowing the rise time, we note that $\kappa$ approaches the predicted interval $0 \leq \kappa \lesssim 68$.

The phenomenon of enhancement in the magnitude of the three-body recombination coefficient gives an indication that may be a laser-like effect is occurring in the experiment of ref. [8]. Our picture suggests that for very short time scales (time ranges varying from 0 up to $100 \mu \mathrm{s}$ ) the three-body recombination processes produce shallow dimers and a third atom (burst effect) in many-boson states. The coherent production of shallow dimers occurs for very short rise times and 
Table 2. Numerical values of the three-body recombination coefficient $\kappa$ as function of the rise time $t_{r}$ of the magnetic field pulses applied to the ${ }^{85} \mathrm{Rb} \mathrm{BEC}$.

\begin{tabular}{c|rrrrrr}
\hline \hline$t_{r}(\mu \mathrm{s})$ & 12.5 & 25.3 & 75.8 & 151.6 & 202.1 & 252.6 \\
\hline$\kappa$ & 1800 & 1700 & 1600 & 500 & 200 & 100 \\
\hline \hline
\end{tabular}

disappears for longer ones, producing the strange effect reported in ref. [8, of a decreasing dissipation from the condensate for long rise times. This effect is brought to the mean-field calculation by the enhanced values of $\kappa$ as a function of $t_{r}$, which can be approximately given by the parameterization

$$
\kappa\left(t_{r}\right)=2300 \exp \left(-0.01 \times \omega t_{r}\right) .
$$

Such parametrization is convenient to study the remaining number of atoms in ${ }^{85} \mathrm{Rb} \mathrm{BEC}$ as a function of the scaled rise time of the applied magnetic pulse, for fixed hold times, with the parameters considered in ref. [8] (where the magnetic field during the hold time is $B_{h}=156.7 \mathrm{G}$ ). The results of our model calculation describes the observed behavior of the remaining number of atoms (See Fig. 3 of ref. [18]).

\section{Conclusions}

In the present communication, we report recent results that we have obtained by considering the three-body recombination processes in ultracold or condensed atomic gases. From the experimental values of the recombination coefficient together with the corresponding two-body scattering length, for atomic systems in a trap, we are able to predict energies of the shallow trimer bound state for a few atomic systems. To obtain the scaling function corresponding to the dependence of the dimensionless recombination parameter with the shallow trimer binding energy, we use a zero range model which is valid for large scattering lengths. We point out that, at least one experimental result for ${ }^{87} \mathrm{Rb}|2,2\rangle$ is outside the interval of theoretical vacuum values of $\kappa$, which may indicate that some particular physics is present in that experiment.

We also observe that, when considering the special situation of fast variations of the two-body scattering length, near a Feshbach resonance, the experimental results can be put consistent with a mean field description, if one allows a strong enhancement of the three-body recombination process motivated by a possible quantum coherence phenomena in the production of dimers (laser effect). However, certainly one has to go beyond the mean-field description with only condensed atoms, to be able to implement the coherence effect in a defined way. Such a mean-field theory should describe simultaneously the dynamics of the condensed atoms, the remaining atoms and the dimers, which is within the scope of a future investigation.

Acknowledgement. This work was supported by Fundação de Amparo à Pesquisa do Estado de São Paulo and Conselho Nacional de Desenvolvimento Científico e Tecnológico. 


\section{References}

1. Efimov, V.: Phys. Lett. B33, 563 (1970)

2. Thomas, L.H.: Phys. Rev. 47, 903 (1935)

3. Frederico, T., Tomio, L., Delfino, A., and Amorim, A.E.A.: Phys. Rev. A60, R9 (1999)

4. Stamper-Kurn, D.M., et al.: Phys. Rev. Lett. 80, 2027 (1998)

5. Burt, E.A., et al.: Phys. Rev. Lett. 79, 337 (1997)

6. Söding, J., et al.: Appl. Phys. B69, 257 (1999)

7. Roberts, J.L., Claussen, N.R., Cornish, S.L., and Wieman, C.E.: Phys. Rev. Lett. 85, 728 (2000)

8. Claussen, N.R., Donley, E.A., Thompson, S.T., and Wieman, C.E.: Phys. Rev. Lett. 89, 010401 (2002)

9. Yamashita, M.T., Frederico, T., Tomio, L., and Delfino, A.: Phys. Rev. A68, 033406 (2003)

10. Yamashita, M.T., Marques de Carvalho, R.S., Tomio, L., and Frederico, T.: Phys. Rev. A68, 012506 (2003)

11. Nielsen, E., Fedorov, D.V., Jensen, A.S., and Garrido, E.: Phys. Rep. 347 (2001) 373, and references therein

12. Fedichev, P.O., Reynolds, M.W., and Shlyapnikov, G.V.: Phys. Rev. Lett. 77, $2921(1996)$

13. Nielsen, E., and Macek, J.H.: Phys. Rev. Lett. 83, 1566 (1999)

14. Esry, B.D., Greene, C.H., and Burke Jr., J.P.: Phys. Rev. Lett. 83, 1751 (1999)

15. Bedaque, P.J., Braaten, E., and Hammer, H.-W.: Phys. Rev. Lett. 85, 908 (2000)

16. Adhikari, S.K., Frederico, T., and Goldman, I.D.: Phys. Rev. Lett. 74, 487 (1995); Adhikari, S.K., and Frederico, T.: Phys. Rev. Lett. 74, 4572 (1995)

17. Borca, B., Dunn, J.W., Kokoouline, V., and Greene, C.H.: Phys. Rev. Lett. 91, 070404 (2003)

18. Filho, V.S., Tomio, L., Gammal, A., Frederico, T.: Preprint cond-mat 0306218

19. Filho, V.S., Abdullaev, F.Kh., Gammal, A., and Tomio, L.: Phys. Rev. A 63, 053603 (2001)

20. Moerdijk, A.J., Verhaar, B.J., and Axelsson, A.: Phys. Rev. A 51, 4852 (1995) 